\title{
Impact of Knowledge about Early Ambulation on Patients' Satisfaction Post Coronary Angiography , at Assiut University Hospital
}

\author{
Mahgoub , A., A ; Mohamed , W., Y ; Mohammed , M ., A; Abdel-Aziz , M ., A \& Kishk , Y ; Taha. \\ Assistant lecture of critical Care Nursing, Assiut University \\ Professor of Critical Care \& Emergency, Cairo University \\ Lecturer in critical Care Nursing, Assiut University \\ Lecturer in critical Care Nursing, Assiut University \\ Professor of cardiology, Faculty of Medicine, Assiut University
}

\begin{abstract}
Coronary Angiography has become a main diagnostic procedure for diagnosis of Coronary Artery Disease (CAD). Aim: this study was carried out1- to investigate the impact of knowledge aboutearly ambulationon patients' satisfaction among post coronary angiography2-early ambulation after 2 hours versus 6 hours.Design: a quasiexperimental design. Setting: in catheterization and coronary care units.Subjects: A convenience sample of all adult educable and mentally competent male and female patients aged from (18-60 years old) who had undergone a nonemergency coronary angiography (CA) through femoral artery during a period from July 2010 to June2011 were eligible for inclusion in the sample.Tools: four tools were utilized to collect data pertinent to the study, tool I assessment of patients after femoral sheath removal and angiography related data tool II: pre/post femoral coronary angiography knowledge sheet toolIIIpre/post femoral coronary angiography observation check list.toolIV satisfaction assessment sheet. Methods: pretest knowledge sheet filled out by the patient and observation checklist sheet was checked by the researcher for both groups ,the teaching protocol has been implemented for patient in terms of session, each session ranged from 6-10 patients for theoretical and practical contents ,then immediately post knowledge sheet test filled out by the patient and observation checklist sheet was checked by the researcher for both groups Results: Finding of the present study revealed that significant improvement of all parameters with values of less than $\mathrm{p}=0.001$ in response to give information among the two groups CA. Conclusion: educating patients before diagnostic cardiac catheterization can effectively improve level of knowledge and satisfaction.
\end{abstract}

\section{Key Words: Early ambulation, Patients' satisfaction, post coronary angiography, patient knowledge}

\section{Introduction}

Coronary artery disease (CAD) is a leading cause of mortality, morbidity, and loss of quality of life globally. Jamshidi etal, 2011.Currently, Coronary angiography has become a main diagnostic procedure for diagnosis of CAD performed in many health care centers .Rezaei et al., 2009.Currently, catheterization has become a routine diagnostic procedure performed in many hospitals. Although it can be performed through brachial, radial, or femoral arteries.Smith et al., 2006. The transfemoral puncture is the most common approach. However, because vascular complications occur in $0.43-5.8 \%$ of transfemoral cardiac catheterization patients, strict bed rest and immobilization of the catheterized leg have been considered essential to reduce the risk of their development.Fraker and Fihn ,2007

Provision of education may equip patients undergoing coronary angioplasty with the knowledge and skills to ameliorate the previously high lighted problems. Aiming to provide sufficient information to enable patients to cope with all aspects and implications of the disease and assume ultimate responsibility for their health care.Education can equip patients with the skills to recognize and alleviate fear and anxiety, to identify and deal with stress-provoking situations and to express the emotional meaning the illness has for them. On its own, education has been shown to significantly decrease cardiac mortality, improve psychological status, improve knowledge and decrease risk factor behaviours in patients after coronary angioplasty.Anderson et al.,2007

Patient satisfaction is considered a marker of effectiveness of health care delivery and is often used as a benchmark of health system performance .Patient satisfaction has been shown to be influenced by physician communication skills, age, patient expectations and length of relationship with the physician. There is increasing interest in the potential influence of a patient's clinical condition on their satisfaction with care .Wenger ,1998

Nurses play an important role in promotion of the patient's knowledge before an invasive procedure such as CA . Due to the lack of knowledge, patients experience anxiety, stress, and consequently hemodynamic instability in response to an invasive CA.Ruffinengo , 2009Furthermore, due to prolonged 
bed rest in a fixed position after the procedure, the patients report feelings of discomfort and intolerance.Chair and Wong,2004 Patient's knowledge may decrease their psychological problems, significantly decrease the nursing work load, reduce the hospital stay, and also promote the patients and nurses' satisfaction, comfort and tolerance related to an invasive procedure . Ayral et al.,2002Many studies have shown that the patients benefit from information about an invasive diagnostic procedure.Phillipe etal 2006There are many methods of patients' education like verbal information, written information, leaflets, booklets, audiotapes, and video information. but an ideal method to be used for patients' education is still unknown. In spite of these methods, the verbal information by nurses and physicians is the common routine for patient's education before CA in many hospitals.Steeenino et al.,2007

\section{Aim of the study}

The aim of this study is to investigate the impact of knowledge about early ambulation on patients' satisfaction post femoral coronary angiography patients. Angiography2-early ambulation after 2 hours versus 6 hours.

\section{Patients and Methods}

\section{Research design}

Quasi experimental research design has been utilized in this study.

\section{Setting:}

This study was carried out in catheterization and coronary care units

\section{Patients:}

A convenience sample of all adult educable and mentally competent male and female patients aged from (18-60 years old) who underwent for nonemergency 6 frensh coronary angiography (CA) through femoral artery were recruited from July 2010 to June 2011.All patients meeting the criteria were approached during the recruitment period. They were assigned into study and control groups.

Inclusion criteria were are considered: All patients had a six French sheath, normal prothrombin time.(10-14 seconds) (Urdenetal 2010),normal renal function test. ( BUN is 5 to $25 \mathrm{mg} / \mathrm{dl}$, creatinine is 0.5 to $1.5 \mathrm{mg} / \mathrm{dl}$ )(Urden etal 2010),hemodynamically stable.

Exclusion criteria were considered, coagulationabnormalities, hypertension, chronic lower back pain, transradial coronary angiographty, groin pathology, previous surgery in the iliac or femoral arteries,therapeutic cardiac catheterization and complications developed during Coronary angiography
Matching criteria were considered,meanagerange of (1-3 years),sex ,the same level of educations(read and write), the same size of sheath $(6 \mathrm{~F})$, the same dose of heparin

Content validity: the tools were tested for content related validity by jury of 5 specialistits in the field of critical care nursing and coronary medicine from Assiut University and Cairo University, and the necessary modifications were done.

Pilot study.Apilot study was conducted on 22 patients after explain the nature and purpose of the study to test the feasibility and applicability of the tools. The necessary modifications are required. These necessary modifications were done and the pilot study patients were excluded from the actual study. The Reliability was done on study tools by cronback's Alpha (0.95)

Protection of human rights:

An Official approval was obtained from hospital administrative authority to collect the necessary data after explanation of the aim and nature of the study. Patients' anonymity and confidentiality were ascertained, patients' was maintained and voluntary participation and right to refuse to participate in the study were emphasized to the patients. Written consent was obtained from patients who are willing to the study.

\section{Study tools}

Three tools were used to collect the data in this study. Patient assessment sheet after femoral sheath removal and angiography related data .This tool consists of two parts and developed by the researcher after review of literature $(5,8,11-13)$ :

Part I: socio- demographic and angiography data which includes:age,sex past medical history, hospital stay and time of homeostasis to assess patient's profile

Part II: assessments after femoral sheath removal, which includes pulse, mean arterial blood pressure and peripheral pulse assessment to assess vital signs and catheterized leg.

Tool II: pre/post femoral coronary angiographyknowledge sheet:

This tool was used to assess and measure the exact patient knowledge about heart function, coronary artery diseases, activity, nutrition, and medication. The same tool was used immediately after the implementation of knowledge about early ambulation (immediately post test). It consists of 30 items covering the previous parts.

Scoring system:

A total score was 60 degree. The 60degree was distributed through its five parts: knowledge about the heart was 10; knowledge about the cardiac catheterization was 20, knowledge about healthy heart diet was 10, knowledge about physical activity 
was 10, knowledge about coronary disease was10.Each right answer was given two degree for all questions with total scores 60 . Those who scored less than $60 \%$ considered as having an unsatisfactory, but more than $60 \%$ considered as satisfactory.

\section{Tool III: Pre/post femoral coronary} angiographyobservational checklist:

This tool was modified by Crven and Hirnle 2008to measure the ability of the patients to move from the bed to chair, chair to walk. The total score was 7 degree .The 7 degree was distributed through seven steps.

Scoring system: patients who were performed the seven steps correctly were considered able to mobilize successfully. A total score of the observational check list was 7 degree. Each right answer was given one degree for all questions with total scores 7. Those who scored less than $60 \%$ considered as having an unsatisfactory, but more than $60 \%$ considered as satisfactory

Tool IV:Satisfaction assessment sheet

This part consists of 12 items short form of the patient satisfaction questionnaire (PSQ).modified by salah etal 2006.(15) The patients had to respond to the questions about both positive negative aspect $\mathrm{s}$ of the procedure on a 3-point scale :"yes a lot"," yes a little", and "no" .These will respectively scored 3,2 and 1 . The higher scores indicate greater satisfaction with the procedure ( $60 \%$ or more), and the lower scores (less than 60\%) indicate dissatisfaction with the procedure and includes procedure related items, patient feeling items, communication.

- Procedure explanation

- Nurse/patient communications

- Comfort

- Procedure time

- No fear from procedure

- No fear from instruments

- Procedure cost

- Doctor checked after procedure

- Lack of anxiety

- Confidence with Procedure

- Lack of stress

- Recommending procedure to others

Procedure

Preparatory phase

- The patient admitted early in the morning at the cardiac catheterization unit.the researcher was obtained demographic and clinical data from the patient's medical record as well as directly from the patient such as the patients' age ,sex,and medical diagnosis.
- Heart rate and mean arterial pressure were measured using bedside monitor connect to the wall by three cardiac electrodes.

- Peripheral pulse was measured by palpitation in the popliteal and dorsal pedis arteries.

\section{Implementation phase \\ For theoretical content:}

-The teaching protocol has been implemented for patients in terms of sessions, with a total of 35 sessions. Number of patients in each session ranged from 6-10 patients. Then the researcher teaching patient using verbal information and then provided each patient $\mathrm{w}$ designed illustrated booklet. The duration of each session was an two hours, including 10 minutes for discussion and feedback .Each session usually started by a summary of what has been taught during this session and the objectives of the topics .Feedback and reinforcement of teaching was performed according to the patients needs to ensure their understanding.

\section{For practical content:}

-The researcher conducting demonstration using teaching materials (photos).This was done on a small sessions ranged from 6-10 patients .The duration of each session was an two hours, including 10 minutes for discussion and feedback.

-Most patients were able to ambulate and can master this skill easily. Then the immediately post practical test was filled out by the researcher.

-Theoretical and practical content were repeated according to the ability of understanding.

\section{Statistical design}

Data was collected and analyzed by computer programmed SPSS (ver.16) .Data were presented using descriptive statistics in the form of frequencies and percentages for qualitative variables, and means and standard deviations for qualitative variables. Qualitative variables were compared using chi-square test to determine significance for non parametric variables-test used to determine significance for numerical variables. The critical value of the tests "P" was considered statistically significant when $\mathrm{P}$ less than 0.05 


\section{Results:}

Table 1: Comparison between the study and control groups in relation to socio demographic data

\begin{tabular}{|c|c|c|c|c|c|}
\hline \multirow{3}{*}{ Socio demographic data } & \multicolumn{5}{|c|}{ Coronary angiography } \\
\hline & Study & $(\mathrm{N}=110)$ & Cont & $\mathrm{N}=110$ ) & \multirow{2}{*}{ P. value } \\
\hline & No & $\%$ & No & $\%$ & \\
\hline $\begin{array}{l}\text { Age group } \\
<40 \text { years } \\
41-50 \text { years } \\
51-60 \text { years }\end{array}$ & $\begin{array}{c}6 \\
34 \\
70 \\
\end{array}$ & $\begin{array}{c}5.5 \\
30.9 \\
63.6 \\
\end{array}$ & $\begin{array}{c}6 \\
40 \\
64 \\
\end{array}$ & $\begin{array}{c}5.5 \\
36.4 \\
58.1 \\
\end{array}$ & 0.686 \\
\hline Mean $\pm S D($ year $)$ & \multicolumn{2}{|c|}{$51.1 \pm 6.7$} & \multicolumn{2}{|c|}{$50.4 \pm 6.2$} & $0.441^{\mathrm{Ns}}$ \\
\hline \multicolumn{6}{|l|}{ Medical diagnosis } \\
\hline Hypertension & 70 & 63.6 & 75 & 68.2 & \multirow{4}{*}{0.731} \\
\hline Diabetes mellitus & 10 & 9.1 & 10 & 9.1 & \\
\hline Previous angina & 30 & 27.3 & 25 & 22.7 & \\
\hline Previous MI & 75 & 68.2 & 70 & 63.6 & \\
\hline
\end{tabular}

Ns: no significant statistical difference $\quad$-Chi-square test - independent samples t-test * significant $a t(p \leq 0.05)$

(Table 1) shows, It noticed that $63.6 \%$ and $58.1 \%$ of the study and control groups were in the age group of 5060 years old,concerning to medical diagnosis, $27.3 \%$ and $22.7 \%$ had previous angina ,there were no significant statistical difference between the two groups in relation to age and medical diagnosis. $(\mathrm{p}=0.686$ and 0.731 respectively)

Table 2: Comparison between the study and control groups in relation to bed rest ,time of compression and hospital stay $($ mean \pm SD)

\begin{tabular}{|c|c|c|c|}
\hline \multirow{2}{*}{ Bed rest , time of compression and hospital stay } & \multicolumn{3}{|c|}{ Coronary angiography } \\
\cline { 2 - 4 } & Study ( N=110) & Control ( N=110) & \multirow{2}{*}{ p.value } \\
\cline { 2 - 4 } & Mean \pm SD & $5.9 \pm 0.61$ & $0.001^{* *}$ \\
\hline Duration of bed rest after CA (hours) & $2.0 \pm 0.01$ & $9.6 \pm 2.5$ & $0.001 * *$ \\
\hline Time of compression to achieve hemeostas is(minutes) & $11.6 \pm 2.9$ & & \\
\hline Hospital stay (\%) & & 100 & NA \\
\hline (less than one day) & 100 & 0 & 0 \\
\hline (more than one day) & 0 & & 0 \\
\hline
\end{tabular}

-Chi-square test $*$ significant at $(p \leq 0.05)$

(Table 2) showsthat, the mean bed rest hours for study and control groups were $\mathbf{2 . 0 \pm 0 . 0 1}$ and 5.9 $\pm \mathbf{0 . 6 1}$ hours with high significant statistical difference between both groups $\mathrm{p}=0.001$. As regards to the mean time of compression to achieve hemeostasis for study and control groups, it was $\mathbf{1 1 . 6 \pm 2 . 9}$ and $\mathbf{9 . 6 \pm 2 . 5}$ minutes with significant statistical difference between both groups $\mathrm{p}=0.001$ Concerning to hospital stay, all patients stayed as the same period of time in study and control groups

Table (3) pre and post information knowledge scores (mean \pm SD)

\begin{tabular}{|l|c|c|c|}
\hline \multirow{2}{*}{\multicolumn{1}{|c}{ Knowledge items }} & \multicolumn{3}{c|}{ Coronary angiography } \\
\cline { 2 - 4 } & Pre test & immediate post test & \multirow{2}{*}{ P.value } \\
\cline { 2 - 4 } & Mean \pm SD( N=110) & Mean \pm SD( N=110) & $0.001 * *$ \\
\hline $\begin{array}{l}\text { Heart and its medication } \\
\text { Maximum score }=10\end{array}$ & $3.98 \pm 2.59$ & $8.73 \pm 1.97$ & $0.001 * *$ \\
\hline $\begin{array}{l}\text { Information before CA } \\
\text { Maximum score }=10\end{array}$ & $1.47 \pm 1.86$ & $7.64 \pm 1.93$ & 0.93 \\
\hline
\end{tabular}




\begin{tabular}{|c|c|c|c|}
\hline \multirow{3}{*}{ Knowledge items } & \multicolumn{3}{|c|}{ Coronary angiography } \\
\hline & Pre test & immediate post test & \multirow{2}{*}{ P.value } \\
\hline & Mean \pm SD $(N=110)$ & $\operatorname{Mean} \pm \operatorname{SD}(\mathrm{N}=110)$ & \\
\hline $\begin{array}{l}\text { Information after CA } \\
\text { Maximum score }=10\end{array}$ & $1.02 \pm 1.36$ & $6.07 \pm 3.03$ & $0.001 * *$ \\
\hline $\begin{array}{l}\text { Nutrition } \\
\text { Maximum score }=10\end{array}$ & $2.47 \pm 1.01$ & $3.02 \pm 0.97$ & $0.001 * *$ \\
\hline $\begin{array}{l}\text { Activity } \\
\text { Maximum score }=10\end{array}$ & $0.31 \pm 0.81$ & $2.04 \pm 1.85$ & $0.001 * *$ \\
\hline $\begin{array}{l}\text { Disease } \\
\text { Maximum score }=10\end{array}$ & $2.44 \pm 1.42$ & $6.91 \pm 2.23$ & $0.001 * *$ \\
\hline $\begin{array}{l}\text { Total scores } \\
\text { Maximum score }=60\end{array}$ & $12.24 \pm 5.39$ & $33.85 \pm 6.26$ & $0.001^{* *}$ \\
\hline
\end{tabular}

Independent t-test $*$ significant at $(p \leq 0.05)$

(Table 3) shows significant improvement of all parameters with values of less than $p=0.001$ in response to give information among the two groups

Table (4) pre and post practice scores among study group (mean \pm SD)

\begin{tabular}{|l|c|c|c|}
\hline \multirow{2}{*}{ Observation check list } & \multicolumn{3}{|c|}{ Coronary angiography } \\
\cline { 2 - 4 } & Pre test & immediate post test & \multirow{2}{*}{ P.value } \\
\cline { 2 - 4 } & mean+SD( N=110) & mean+SD( N=110) & \multirow{2}{*}{$0.001 * *$} \\
\hline $\begin{array}{l}\text { Steps of ambulation } \\
\text { Maximum score }=10\end{array}$ & $6.47 \pm 1.41$ & $9.13 \pm 1.45$ & \\
\hline
\end{tabular}

Independent samples t-test $*$ significant at $(p \leq 0.05)$

(Table 4) shows that, there is alow base line (pre-test) mean scores of practice parameter. However, the post implementing showed a significant improvement with values of $(\mathrm{p}=0.001)$ among two groups.

Table (5): Comparison between the study and control groups in relation to satisfaction score

\begin{tabular}{|c|c|c|c|c|c|}
\hline \multirow{3}{*}{ Satisfaction score } & \multicolumn{5}{|c|}{ Coronary angiography } \\
\hline & \multirow{2}{*}{\multicolumn{2}{|c|}{\begin{tabular}{c|c} 
Study & $(\mathbf{N}=110)$ \\
No & $\%$ \\
\end{tabular}}} & \multicolumn{2}{|c|}{ Control $(\mathrm{N}=110)$} & \multirow[b]{2}{*}{ P. value } \\
\hline & & & No & $\%$ & \\
\hline \multicolumn{6}{|l|}{ Procedure relation } \\
\hline -Procedure explanation & 110 & 100 & 70 & 63 & $0.001 * *$ \\
\hline -Procedure time & 98 & 89 & 40 & 36 & $0.001 * *$ \\
\hline -Procedure cost & 78 & 71 & 100 & 91 & $0.001 * *$ \\
\hline -Confidence with Procedure & 92 & 83 & 88 & 80 & 0.299 \\
\hline \multicolumn{6}{|l|}{ Patient feeling } \\
\hline -Lack of anxiety & 90 & 82 & 83 & 75 & 0.161 \\
\hline -Comfort & 95 & 86 & 94 & 85 & 0.501 \\
\hline -No fear from procedure & 99 & 90 & 31 & 28 & $0.001 * *$ \\
\hline -No fear from instruments & 100 & 91 & 31 & 28 & $0.001 * *$ \\
\hline -Lack of stress & 80 & 73 & 50 & 45 & $0.001 * *$ \\
\hline \multicolumn{6}{|l|}{ Communications } \\
\hline -Nurse/patient communications & 90 & 82 & 80 & 73 & 0.073 \\
\hline -Doctor checked after procedure & 88 & 86 & 95 & 86 & 0.139 \\
\hline -Recommending procedure to others & 80 & 73 & 50 & 45 & $0.001 * *$ \\
\hline Score $($ Mean \pm SD $)$ & \multicolumn{2}{|c|}{$83.7 \pm 13.1$} & \multicolumn{2}{|c|}{$79.9 \pm 14.6$} & $0.045 *$ \\
\hline
\end{tabular}

NA not applicable Chi-sqare test* significant at $(p \leq 0.05 \quad$ independent test

(Table 5)show that significant statistical differences between two groups in mean score $\mathrm{p}=0.045$ 


\section{Discussion:}

The aim of this study was to investigate the impact of knowledge about early ambulation among post coronary angiography on patients' satisfaction. The present study presented that the majority of both groups were in age group 50 to 60 years old and most patient were males .This can be attributed to the higher exposure to life stress, and female hormones protect female from CAD. This in line with Andrea, 2010 who studied early sheath removal and ambulation in patients submitted to cardiac catheterization : A randomized clinical trial, found that $64 \%$ of the study sample was males and their mean age were $59.7 \%$ years old. This is may be related to changes of the heart and blood vessels that occur with aging such as the decrease in elasticity and the ability to respond to changes in compliance of the arterial system that increase the work needed to drive the blood to the various organs of the body due to resultant increase in the resistance to the pumping action of the heart.(Jung et al., 2009).

The presented study show that less than half of the control and study groups of patients undergoing CA had hypertension and diabetes mellitus.Abdel -El Ghany,2009 agree with current study reported that $36.7 \%$ of the study had hypertension and $49 \%$ had diabetes mellitus.

Management of hemeostasis at the access site after cardiac catheterization is important to reduce complications, increase patient comfort and safety, and decrease hospital stay. Management of the arterial access site after diagnostic catheterization continues to evolve. The current study found that $100 \%$ of study and control groups in CA using manual compression. Ford, 2008 agree with current study who mentioned that manual compression is the "gold standard" after femoral arterial sheath removal.This may attributed other vascular closure devices contribute to increase health care cost, and it remains unclear whether closure devices reduce vascular complication rates . Also, Boztosun et al.,2008 documented that manual compression hemeostasis followed by bed rest has been the standard of care following cardiac catheterization via femoral access.

Prolonged bed rest is one of the conservative measures to avoid local bleeding but is associated with discomfort Lunde et al., 2006. The current study show that the mean bed rest hours for control and study groups were $5.9 \pm 0.6$ and $2.0 \pm 0.01$ hours .Chair et al.,2008reported that the duration of the bed rest varies from 2 to 24 hours and there is no recommendation on the optimal duration of such a bed rest .Mc cabe et al., 2001 documented that prolonged bed rest in supine position is based on the previous nursing experience rather than on research Many studies focus on reducing bed rest duration during the post-coronary procedures in an attempt to promote patient's comfort. Among the studies reviewed, some studies such as those conducted by Vlasic et al,2004 as actually involved alternative interventions such as elevation of the head-of-bed and changing the patient's position during bed rest. The effect of changing position has been further examined by Chair et al,2003in a randomised controlled study with fairly large sample size of 419 Chinese patients undergoing cardiac catheterization.

The current study findings revealed that the study group was more satisfied about early ambulation than control groups.In this respect. Patient satisfaction can drive patients to facilities where the most progressive care is provided, such as femoral access catheterization. Assisting the patients' understanding of the benefits of femoral access catheterizations will reduce anxiety and provide a more positive experience. Information about patient satisfaction is valuable, especially when there is a question about the alternate access site, but nurses and technologists also need to understand the complications associated with transradial catheterization so they can anticipate and try to prevent them.Significantly decrease the nursing work load,reduce the hospital stay, and also promote the patients' satisfaction, comfort and tolerance related to an invasive procedure.

In addition, many studies have shown that patient satisfaction results were often skewed to the positive responses, especially with older patients, and the instruments used may not be sensitive enough to detect differences between groups of patients Chair et al., 2003 Therefore, the results of this study should be interpreted cautiously.

McDonnell,1999 found conflicting evidence concerning providing pre procedural information, which is in line with the lack of consistent evidence for using decisional aids culminating in conflicting recommendations. Astley et al.,2008 conducted a randomized control trial using various decisional aids, with verbal patient education sessions considered 'usual care'. These investigators found no difference in recall, patient satisfaction or anxiety levels. Regardless of these findings, the legal requirements for obtaining informed consent remain an important consideration.

Clark et al.,2005 emphasise the need for health care providers to seek regular feedback regarding the quality of pre-discharge education. Given the diverse patient characteristics in relation to health literacy, English comprehension and level of education, appropriate forms of information sharing are required 
to improve access to and retention of the information shared. Evidence of long-term effectiveness of secondary prevention strategies is still emerging.In a 10-year follow-up study of nurse led secondary prevention programs in primary care settings, Delaney et al.,2008 concluded that the closer to diagnosis secondary prevention strategies are employed the greater the chance patients have for medium to long-term survival. Nurses play an important role in advocating for secondary prevention strategies with patients and family

Another most important finding of this study was that information was effective in improving the level ofcomfort and satisfaction. This impliesthat the levels of comfort, satisfaction might be related to the knowledge of patients based on information. This result is similar to that of Phillipe et al., 2006, showed that information before the procedure of cardiac catheterization increases the tolerance of the procedure by patients. In the study by Ayral et al., 2002, patients who had knowledge and information reported significantly better tolerance (91\% vs. $48 \%$ ) than the control group. One of the most important findings was that patients in the experimental group were more significantly satisfied than the control group.

Previous studies revealed that watching an informational video before an invasive procedure like CA not only decreases the patient's anxiety, but also improves their tolerance and satisfaction Obled et al., 2007. The level of comfort in the experimental group was significantly higher than the control group. Rezaei,2009 showed that patient's education about changing position inbed can effectively maximize his/her comfort. It is concludedthat orientation of the patients by informationabout the procedure as well as care before, during and afterCA increases the tolerance and comfort of patients.

Early ambulation was not shown to have an effect on improving patients' satisfaction level, which was not consistent with the previous study Basuny, 2009 Cultural influences should be taken into consideration when assessing the satisfaction level of patients because culture influences how feelings are expressed and what verbal and nonverbal expressions are appropriate Vlasic et al.,2004.feelings about the care they received would disturb the harmony.

Nurses on the front line caring for patients before, during and after cardiac catheterization play a key role in the prevention of complications. With the increasing number of cardiac catheterizations performed, evolving technology, and advances in pharmaceutical therapy comes an increase risk of vascular complications.Andrea et al.,2010

Finally, teaching patient can effectively improve patient knowledge and satisfaction.

\section{Conclusion and Recommendations}

Based on the findings of the present study, it can be concluded educating patient before diagnostic cardiac catheterization via femoral artery access can effectively improve level of knowledge and satisfaction.

In the light of the above, the following recommendations are suggested:-

- Equip the cardiac catheterization unit with simple illustrated guidelines protocol covering the major early ambulation practices post femoral cardiac catheterization

- Develop an in service audiovisual materials training about early ambulation, complications and its management after cardiac catheterization for patients.

- Establishing a standardized protocol for early ambulation after diagnostic and therapeutic cardiac catheterization.

- Apply clinical practice guidelines for optimal patient care after femoral cardiac catheterization.

- Early ambulation should be added to the routine care.

- Using other methods of education before cardiac catheterization

- Educate patients who are undergoing to cardiac catheterization the day before procedure.

\section{References:}

1. Jamshidi, N., Abbaszadeh, A., \& Kalyani, M. N. (2013). Effects of video information on anxiety, stress and depression of patients undergoing coronary angiographyCollegian $\mathbf{2 0}$, $153-159$

2. Rezaei-Adaryani, M., Ahmadi, F., \& AsghariJafarabadi, M. (2009).The effect of changing position and early ambulation aftercardiac catheterization on patients' outcomes: A singleblindrandomized controlled trial. International Journal of NursingStudies, 65(2), 417-424

3. Smith SC, Feldman TE, Hirshfeld JW, Jacobs AK, Kern MJ, King SB, 2006. ACC/AHA/SCAI 2005 Guideline update for percutaneous coronary intervention summar . Circulation; 113:e166-286

4. Fraker TD Jr, Fihn SD. (2007) Chronic Unstable Angina Working Committee. Chronic angina focused update of the ACC/AHA 2002 guidelines for the management of patients with chronic unstable angina: A report of the American College of Cardiology/American HeartAssociation Task Force on Practice Guidelines Working Group to develop the focused update of the 2002 guidelines for the 
management of patients with chronic stable angina. J Am Coll Cardiol;4: 2264-74.

5. Anderson JL, Adams CD, Antman EM, Bridges CR, Califf RM, Casey DE Jr, . ACC/AHA 2007 guidelines for the management of patients with unstable angina/ non STelevation myocardial infarction executive summary: a report of the American College ofCardiology/American Heart Association Task Force on Practice Guidelines (Writing Committee to revise the 2002 guidelines for the management of patients with unstable angina/non ST-elevation. J Am Coll Cardiol;50:652-726.

6. Wenger NK.( 1992) Patient and family education and counsel ing: $\mathrm{A}$ requisite component of cardiac rehabilitation. In Mathes $\mathrm{P}$, Halhuber MJ, editors. Controversies in car diac rehabilitation. Berlin: Springer-Verlag,

7. Ruffinengo, C., Versino, E., \& Renga, G. (2009). Effectiveness of an informative video on reducing anxiety levels in patients undergoing elective coronarography: An RCT. European Journal of Cardiovascular Nursing, 8, 57-61.

8. Chair, S. Y., Li, K. M., \& Wong, S. W. (2004). Factors that affectback pain among Hong Kong Chinese patients after cardiaccatheterization. European Journal of Cardiovascular Nursing, 3, $279-285$

9. Ayral, X., Gicquere, C., Duhalde, A., Boucheny, D., \& Dougados,M. (2002). Effects of video information on preoperative anxiety level and tolerability of joint lavage in knee osteoarthritis. Arthritis and Rheumatism, 47(4), 380-382

10. Phillipe, E., Meney, M., Larrazet, F., BenAbderrazak, F., Dibie,A., Meziane, T., et al. (2006). Effects of video information inpatients undergoing coronary angiography. Archives des Maladies du Coeur et des Vaisseaux, 99(2), 95101

11. Steffenino, G., Viada, E., Marengo, B., \& Canale, R. (2007).Effectiveness of video-based patient information before percutaneouscardiac interventions. Journal of Cardiovascular Medicine, 8(5)20-33

12. Crven C., Hirnle A (2008): Fundemental of medical surgical nursing.Mosby,INC,282-288

13. Salah M,SaidH,Mohammed H,Okasha $N$.Comparison of the effectiveness of three groin compression methods following cardiac catheterization.SC.J.AZ.Med.fac(girles)27(3), 20 06:1547-1566.

14. Andrea, K., Bregendahl, M., Kaestel, H.,. (2010): Early sheath removal and ambulation in patients submitted to percutaneous coronary intervention: A randomised clinical trial International Journal of Nursing Studies 47 939945. www.elsevier.com/ijnsbased? Images 2003 Fall;22(3):9-12.

15. Jung U, Torrejon C, Tigh A .( 2009): N-3 fatty acids and cardiovascular diseease :mechanism underling beneficial effects. The American $\mathbf{J}$ of clinical nutrition 87-1992-1499

16. Basuny S. (2009): The effect of positioning changing post coronary angiography on patient,s outcomes,Master thesis, Faculty of Nursing,Alexandria university

17. Abdel-Ghany A.(2009):Theraputic value of PTCA in early stages of acute MI.Doctoral thesis ,Faculty of Medicine,Assiut University.

18. Ford,S(2008):DETERMINING BEST PRACTICE: REMOVAL OF FEMORAL ARTERIAL SHEATHS. Doctor of Nursing, University of South Carolina

19. Boztosun B, Guneş Y, Yıldız A, Bulut M, Săglam M, Kargın R, et al.( 2008): Early ambulation after diagnostic heart catheterization. Angiology; 58: 743-6. catheterization on patient outcomes. J Clin Nurs; 16: 212-4.

20. Lauck S, Johnson J, Ratner P.( 2006 ): A Selfcare behaviour and factors associated with patient outcomes following same-day discharge percutaneous coronary intervention European Journal of Cardiovascular Nursing (8): 190-199.

21. Chair, S, Thompson, D.( 2008): The effect of ambulation after cardiac catheterization on patient outcomes. Journal of Clinical Nursing 16, 212-214.

22. LB, Pieper KS. (2011): Vascular complications of cardiac catheterization. J Vasc Surg;14:375381

23. Vlasic W.( 2004): An evidence-based approach to reducing bed rest in the invasive cardiology patient population. Evid Based Nurs; 7: 100-1.

24. Chair, S.Y., Taylor-Piliae, R.E., Lam, G., Chan, S. (2003): Effect of positioning on back pain after coronary angiography. Journal of Advanced Nursing 42 (5), 470-478.

25. McDonnell A.( 1999) A systematic review to determine the effectiveness of preparatory information in improving the outcomes of adult patients undergoing invasive procedures. Clin Eff Nurs.;3(1):4-13

26. Astley CM, Chew DP, Aylward PE, Molloy DA, De Pasquale CG. (2008).A randomized study of three different informational aids prior to coronary angiography, measuring patient recall, satisfaction and anxiety. Heart Lung Circ.;17(1):25-32 
27. Clark PA, Drain M, Gesell SB, Mylod DM, Kaldenberg DO, Hamilton J.( 2005) Patient perceptions of quality in discharge information. Patient Educ Couns.;59:56-68

28. Delaney EK, Murchie P, Lee AJ, Ritchie LD, Campbell NC. (2008).Secondary prevention clinics for coronary heart disease: a 10 year follow-up of a randomised controlled trial in primary care. Heart.;94:1419-1423 